\title{
Key Technology Research on the Establishment of Image Database
}

\author{
Tao Duan ${ }^{1,}$, , Jing Huang ${ }^{1, b}$ \\ ${ }^{1}$ Henan University of Chinese Medicine, ZhengZhou, Henan, China, 450046 \\ a email, ${ }^{\text {b email }}$
}

\begin{abstract}
Keywords: Key Technology, Image Database, Image Coding
\end{abstract}
\begin{abstract}
The establishment of a powerful image database system is a high effective method of the organization and management of massive image data. This paper firstly analyzes the key technology of image database establishment, including section technology, construction technology and query technology of image data, and then gives the design and implementation methods of the image database system to provide some references for the related researchers.
\end{abstract}

\section{Introduction}

With the improvement of the digital degree of aviation and aerospace photogrammetry field, the results of all kinds of digital images are becoming more and more abundant. The expansion of the scale of the digital image data has caused the image data storage and backup, retrieval, and the use of time and effort. Image database management system to meet the needs of massive image data preprocessing, data storage, management, query and browse, distribution, sharing and other aspects of the demand, so the establishment of this system has strong forward-looking and necessity. Database system must introduce new technology to meet the requirements of the application of data processing. In order to efficiently manage such as graphics, images, audio, spatial audio and other non-standardized data, several major database vendors have begun to transform and expand their own concept of relational database, multimedia data type and object oriented. Oracle company in its own system to join the Ware Spatial component to support spatial data. Parallel database technology and distributed database technology will provide powerful technical means for the management of complex mass data. The parallel processing of the database is an effective way to improve the ability of quick response to the transaction database system, which is composed of a plurality of processor to finish the task by collaborative methods a database partition the task into multiple sub tasks, which can greatly improve the ability of transaction processing, the database performance is greatly improved. The distributed database system is composed of a plurality of computers, each of which is provided with a local database and a communication network connection. In distributed system, the data is managed by the system, so that the user doesn't feel the distribution of the data, and the user can see the centralized database of a global model. The architecture of distributed database system is flexible, the system reliability is high, and the availability and extensibility of the system are good. From the current computer technology, network communication technology, mass data storage technology, computer software technology and database technology development, for the establishment of large-scale image database has laid a solid technical foundation. Therefore, from the perspective of the development of related technologies, it is feasible to establish a large image database. 


\section{Key Technology of the Establishment of Image Database}

Section Technology of Image Data. Due to the massive image database, the image data is only a small part of the database, if the data file is very large, it will directly affect the speed of data reading. It is the key technology of the image database to organize and manage the data efficiently, and the data block. In simple terms, there is no general standard to follow. It can be divided into arbitrary rules, but in the application will have to consider some practical problems. Data blocks too large or too small will affect the system's performance. If the data block is too large, it may result in excessive reading of the data. The image data is an attempt to increase the production units, each map as the file to store the final product. The picture itself is a rule of the whole geographical space division. In fact, every map file can be viewed as a block, but because each picture is still a great amount of data, the image storage, scheduling, management and application are very bulky, low efficiency. As a result, the internal data of the figure should be divided again. At present, there are two ways to divide the image blocks. Bands can be sequentially encoded and can be retrieved separately. This partitioning method has been used in several data formats. The method is to divide massive image data in the grid is divided into small blocks, usually square, sometimes also rectangular. There are two main ways to retrieve the spatial data: one is according to the geographical scope. The system should be based on user specified geographic area, such as longitude, Gauss coordinate range and map editing. The scope of administrative divisions of the administrative divisions and other related information; the other is in accordance with the spatial entity object, according to the attributes of the spatial object, such as the name of the river and the code, the name of the road and the coding. For example, this paper uses the establishment of place name database. According to the attributes of spatial objects, fast and accurate image data are determined. Some data formats also support this partitioning method. Compared to the zonal division, the block partition is more suitable for image indexing and mosaic, and the partition of the block has good clustering characteristics. This method is used to partition the data blocks in the image database.

Construction Technology of Image Data. The construction method of the most commonly used image data is Pyramid construction method. Image Pyramid structure refers to the reference in the same space, according to the needs of the user can be stored and displayed in different resolution, the resolution formed from low to high. The Pyramid image structure for image encoding and progressive image transmission is a typical form of hierarchical data structure, multi resolution organization for raster data and image data, is also a kind of raster data or image data compression. In the Pyramid structure, the image is represented by a hierarchical representation. At the top of the Pyramid structure, the lowest resolution data storage; with the increase of the number of Pyramid, the resolution of the data is reduced; at the bottom of the Pyramid, the storage can meet the needs of users of the data of the highest resolution. Each layer is equivalent to a reduction of the resolution of the image estimation. Image Pyramid, as its name suggests, is based on the original image to generate different scales of each layer of image data, and each layer is the same size of multiple slice files to save. The server in dealing with the user's request, the first positioning data to a layer closest to the scale, and then check out the image slice coverage range of user requests and return to the client, and then finally complete splicing is presented to the user on the web page. In the process, only a small amount of data to the server and transfer the user interest to the client, and does not require real-time computation is generated, so the response speed is fast, almost can achieve real-time zoom. Image Pyramid construction algorithm can be likened to a processing plant, the input is the original image data, the output of the Pyramid section of the document. Different algorithms, the final output may be exactly the same, but due to the middle of the processing 
method is not the same, then the processing efficiency, operability, flexibility, etc. there will be a lot of difference.

Query Technology of Image Data. In the image data query, query object is generally rectangular area image geography coordinates, by region size and user viewport size calculated ratio, and then can get the image data to be extracted in Pyramid which, at the level of the corresponding map through a hash function can be grid query geographical coordinates the mapping range blocks to get the i-number, and intersecting the query range all the blocks. Remote sensing data cataloging is the organization and arrangement of remote sensing data according to certain rules, and it will be edited into a directory for people to retrieve data. It is mainly based on the image metadata and image data through field entity one-to-one association, through the image data only to find the image metadata entity, and can only find the image through the image metadata entity data corresponding to the query, so that through the image catalog data can achieve the purpose of information query image data. Core metadata for image coding is to improve the query efficiency of mass image data based on the Landsat three satellite system application requirements, to extract the core metadata set, and then through the core metadata of image data, named unified file, with file name as the only sign associated image data and entity image metadata, and then image core image metadata cataloging for users to query database. The image data of the tuple catalogue and archive based on the image core metadata attribute data and image browsing image as a tuple in the form of unified storage of relational database tables, as a whole for storage and management, which can enhance the integrity and consistency of image data, reduce the query cost. At the same time, we can make the image cataloging system as the general relation database query, delete, update and other operations, image core metadata. Image catalog management is the unified management of image catalog data according to the core metadata.

\section{Function Design and Realization of Image Database System}

Function Design of Image Database System. Image database management system is based on the database technology as the core, integrated image preprocessing, hierarchical storage management, distributed network services and other technologies to achieve high performance software products and more remote platform, multi sensor and multi temporal multi scale image data management and application, can satisfy the mass image data preprocessing, data base, organization management, query and browse, distribution, sharing and other aspects of demand. The main business functions of image database management system include the following. Image database to meet the needs of different image data types and data formats, to provide a task in the way of multiple types of image data library. The database provides a variety of data query methods, including simple query, node query and spatial query. Image database provides a variety of ways of statistical analysis, including the whole library statistics, distribution statistics and storage statistics. Image database provides the management of directory tree, data type, storage node, recycle bin, as well as space field configuration, metadata field configuration, image migration and backup recovery. The image database provides the configuration function of the whole system, including the unified management and maintenance of users, roles, permissions, database configuration, directory tree management and system log. Image database provides data preprocessing functions, including metadata extraction, metadata acquisition and fast view collection.

Function Realization of Image Database System. After the completion of the system background database deployment, you can configure the database and query distribution according to the data situation. Image database will be the original data for the extraction of metadata information, fast view collection; on this basis, based on the extraction of metadata information for 
the formation and configuration of metadata tables. The preparation work includes data modeling, configuration storage nodes and directory tree, data modeling and configuration storage nodes in order, but only after data modeling and configuration storage nodes can the directory tree. In the process of modeling, according to the needs of different types of file storage format and the specific image data to establish the corresponding model for the data type; format is particularly complex, various types of image data files, can establish the packet type. The above work is completed in the case of data node selection and image data corresponding to the data storage library, after the success of the quick view can view on the storage of images, and can be pulled through image frame, artificial interaction, comprehensive query method, and can realize the automatic generation and distribution orders. Function authority is the user's right to have the system function. System installed in a number of built-in functions, such as system management, data preprocessing, storage, data distribution. You can also create and configure a certain kind of authority role, and give the right to use the function of the system. Data authority refers to the user's rights to image data. Authority management is the concept of data through the data role to achieve, can be understood as the data and the synthesis method of these two kinds of user authority management based on the new data character, and then specify the data node of the role can access and have the permission by the user. But the test staff that this management method in a large number of users and data is the need to build a lot of data, but will increase the difficulty of management, based on the user's rights management seems to be easier to understand, at present the privilege management system can achieve. The image library provides the system log function is very detailed, each user exit from the landing, in which all operations of the system and data, will be detailed record for system administrator in the background check.

\section{Conclusion}

The establishment of the image database system can greatly enhance the ability of image data management and application services. The system can provide data support for the comprehensive ability of image service. The key technology of image database will be applied in various industries in the future.

\section{Acknowledgements}

This research was financially supported by the Foundation of Research project of "educational and teaching reform" in Henan University of Chinese Medicine in 2015 (Grant No. 2015JX23).

\section{References}

[1] Li Deren, Wang Mi, Pan Jun, Hu Fen, Geomatics and Information Science of Wuhan University, Vol. 32 (2007) No 11, p.950-954

[2] Gao Xiang, Xu Zhu, Science of Surveying and Mapping, Vol. 41 (2016) No 2, p.121-125+162

3] Guo Huayuan, Xue Wanguo, Yin Ling, Du Peng, Journal of Biomedical Engineering, Vol. 30 (2013) No 1, p.22-27

[4] Wu Chunxia, Digital Technology and Application, Vol. 34 (2016) No 6, p.236 